\title{
A silicon micromachined microphone for fluid mechanics research
}

\author{
Chunchieh Huang ${ }^{1}$, Ahmed Naguib ${ }^{2}$, Elias Soupos ${ }^{3}$ \\ and Khalil Najafi ${ }^{1}$
}

${ }^{1}$ Center for Wireless Integrated MicroSystems, University of Michigan, 1301 Beal Avenue,

Ann Arbor, MI 48109-2122, USA

${ }^{2}$ Mechanical Engineering Department, Michigan State University, East Lansing,

MI 48824-1226, USA

${ }^{3}$ Mechanical Materials and Aerospace Engineering Department, Illinois Institute of

Technology, Chicago, IL 60616, USA

Received 19 April 2002, in final form 9 August 2002

Published 5 September 2002

Online at stacks.iop.org/JMM/12/767

\begin{abstract}
MEMS piezoresistive sound detectors have been fabricated using the dissolved wafer process for the first time. The sensors utilize stress compensated PECVD ultra-thin silicon-nitride/oxide membrane together with monocrystalline ion-implanted $\mathrm{p}^{++}$silicon piezoresistors to achieve high sensitivity. Tests reveal that sensors with a diaphragm size of $710 \mu \mathrm{m}$ have a static sensitivity of $1.1 \mu \mathrm{V} \mathrm{VPa}^{-1}$ with $2 \%$ non-linearity over an operating pressure range of $10 \mathrm{kPa}$. This sensitivity is substantially larger than that of commercially available microfabricated sensors. Furthermore, the new sensor's dynamic response is found to be flat (within $\pm 2.5 \mathrm{~dB}$ ) over a frequency range extending up to $10 \mathrm{kHz}$. This paper contributes to existing literature in the field by demonstrating a new way of fabricating capable MEMS piezoresistive pressure sensors, hence adding to the overall versatility of the technology and associated range of applications.
\end{abstract}

(Some figures in this article are in colour only in the electronic version)

\section{Introduction}

Sound detectors or microphones are transducers that convert acoustical energy into electrical energy. Many transduction schemes have been developed over the years to achieve such conversion, including piezoelectric, capacitive, FET and piezoresistive [1]. The current paper describes the development and testing of piezoresistive silicon sound detectors for measurement of the sound field at the lip of a jet during supersonic jet screech [2, 3]. Ultimately, an array of these detectors is to be integrated with microactuators to implement a feedback control algorithm aimed at reduction/cancellation of screech noise. For more information on jet screech, its physical nature, and associated sound pressure levels (SPLs) and frequencies, the reader is referred to Tam [4].

A piezoresistive microphone consists of a diaphragm that supports four piezoresistors in a Wheatstone bridge configuration. These resistors are located at the edge of the diaphragm with two of them aligned radially and two tangentially to the diaphragm ${ }^{4}$. When the diaphragm deflects under the action of the unknown pressure, the strain along the edge of the diaphragm causes an opposite resistance change in the radial and tangential piezoresistors, which superpose to produce a proportional bridge output.

Recently, a few efforts have been aimed at realizing microfabricated piezoresistive microphones for fluid mechanics applications [5], but the sensitivity has been small (less than $0.1 \mu \mathrm{V} \mathrm{VPa}^{-1}$ for a device with $100 \mu \mathrm{m}$ square diaphragm). Larger devices have been fabricated with improved performance. Schellin et al have measured a sensitivity of $4.17 \mu \mathrm{V} \mathrm{VPa}^{-1}$ with a piezoresistive microphone that consists of $1 \mu \mathrm{m}$ thick, highly boron-doped silicon diaphragm with an area of $1 \mathrm{~mm}^{2}$ and four $0.25 \mu \mathrm{m}$ thick p-type polysilicon resistors [6]. Sheplak et al constructed a silicon-based microphone with a piezoresistive sensing scheme for use in wind tunnel tests [7]. The primary sensing element of the microphone was a $1.5 \mu \mathrm{m}$ thick, $210 \mu \mathrm{m}$ diameter silicon-nitride membrane. On top of the membrane,

4 For p-type piezoresistors in the (100) plane. 


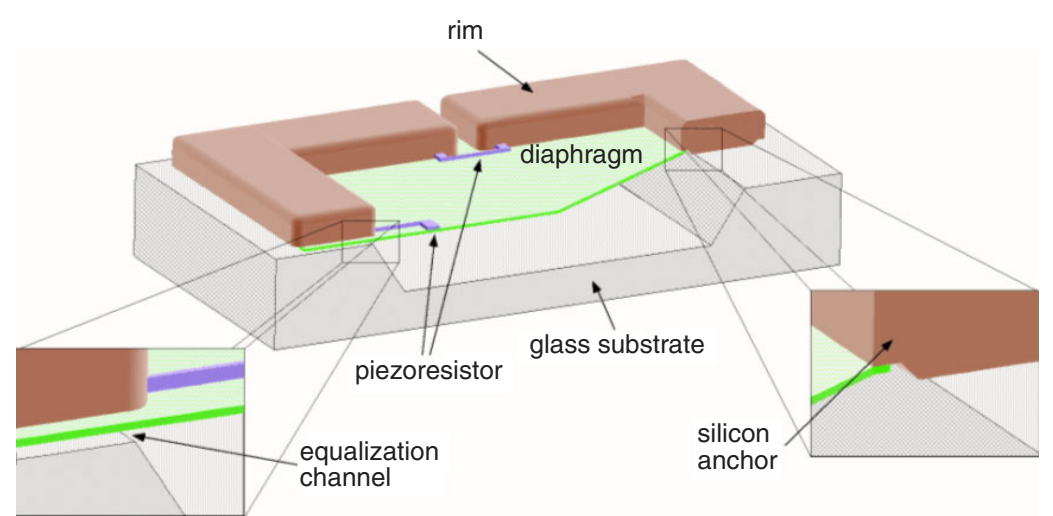

Figure 1. Cut-out view of a 3D model of the piezoresistive sound detector.

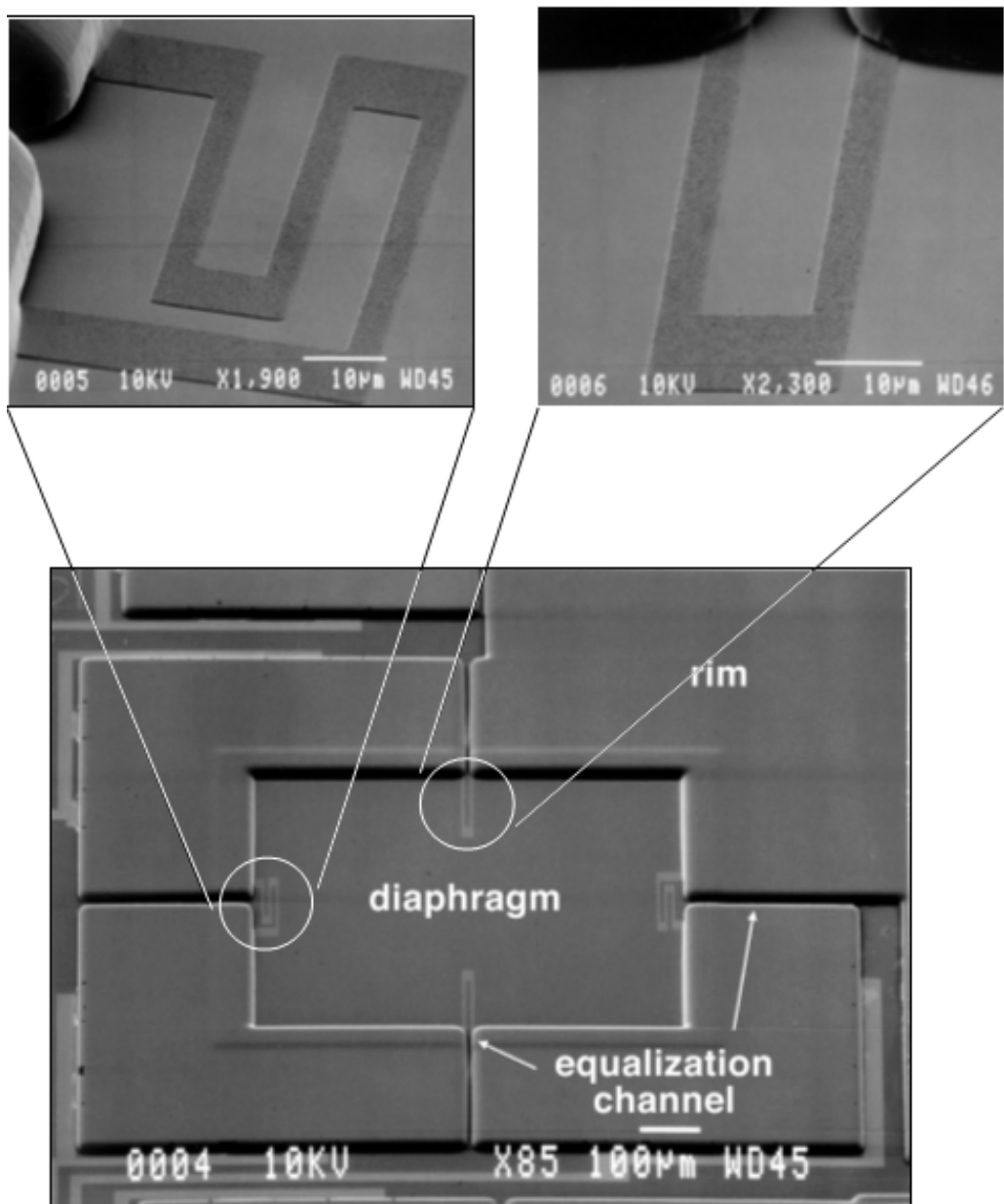

Figure 2. SEM view of a $710 \mu \mathrm{m}$ square silicon sound detector and close-up views of the radial and tangential piezoresistors.

$850 \AA$ thick single-crystal silicon piezoresistors were used in half or full bridge configuration for detection of the diaphragm strain under the action of the measured sound-field pressure. The sensitivity of the microphone was $2.22 \mu \mathrm{V} \mathrm{VPa}^{-1}$ and was flat, to within $3 \mathrm{~dB}$, from $200 \mathrm{~Hz}$ up to at least $6 \mathrm{kHz}$.

A piezoresistive readout scheme is chosen in the current research because of several reasons:

1. the sound level of jet screech is so high $(>120 \mathrm{~dB}$ SPL) that the generally low sensitivity of a piezoresistive readout does not compromise the performance of the sound detectors;
2. the fabrication and readout of a piezoresistive sound detector are much simpler than those of either piezoelectric or capacitive microphones;

3. the fabrication process is compatible with the microactuators to be integrated with the sensors;

4. the bandwidth of a piezoresistive device is not affected by air damping typically encountered in a capacitive device with a small air gap; and finally

5. the current application does not require a wide and flat frequency response over the entire audio frequency range, as is typically needed in an audio microphone. This is due 
to the screech frequency being located within a narrow frequency range.

To compensate for the lower sensitivity of the piezoresistive readout, an ultra-thin diaphragm and ultra-thin monocrystalline highly boron-doped piezoresistors have been developed using a fabrication technology compatible with that of the microactuators [3]. Figure 1 shows a three-dimensional cut-out in the structure of the sound detector developed here. The device consists of a $0.38 \mu \mathrm{m}$ thick two-layer dielectric diaphragm on top of a 'large' $\sim 20 \mu$ m deep cavity. The static (zero-frequency) pressure is equalized across the diaphragm using an equalization channel which runs below the side of the diaphragm and connects to a venting slit in the silicon anchor surrounding the diaphragm. An inset in figure 1 reveals the details of the venting channel.

A SEM view of the actual device is provided in figure 2 . As depicted in the figure, four $2000 \AA$ thick p-type shallow boron diffusion resistors are arranged on the edge of the diaphragm. The specific geometry of the piezoresistors may be seen from the insets in the figure. Two of the piezoresistors are used to measure the radial, while the other two are oriented to measure the tangential (azimuthal) stress. These resistors have the piezoresistance component, $\pi_{44}$, of $70 \times$ $10^{12}\left(\mathrm{~cm}^{2} \mathrm{dyn}^{-1}\right),{ }^{5}[8,9]$, which is about three times higher than that of p-type polysilicon piezoresistors. Therefore, this sound detector is expected to be more sensitive than a polysilicon piezoresistive sound detector. Moreover, the use of single- crystal silicon is expected to result in better $1 / f$ noise characteristics than polycrystalline silicon. Finally, it is worth noting that in general piezoresistive devices suffer from rather large temperature sensitivity [10]. However, as the doping concentration in the resistor increases, the temperature coefficient of sensitivity decreases.

In the next section, the details of the fabrication sequence are presented. This is followed by results of the characterization of the static and dynamic response of the sensor. It should be noted that this paper is the first to describe the fabrication of a piezoresistive MEMS pressure sensor based on the dissolved wafer process [11]. This fabrication sequence is more complicated than that employed in other investigations dealing with the development of similar sensor types. The need to use a more elaborate process here stems from the requirement to make the process compatible with the fabrication sequence of the on-chip actuators mentioned before. In this respect, the current paper adds to existing literature in demonstrating a new way of fabricating MEMS piezoresistive pressure transducers, which in turn adds to the overall versatility of the piezoresistive sensor technology and associated range of applications.

\section{Modified dissolved wafer process for sound detector fabrication}

In order to fabricate the sound detector, some modifications on the original dissolved wafer process [11] are necessary. Figure 3 shows the modified process, which involves eight masks (including one mask to form the microactuator structure). The process starts by recessing the silicon wafer

\footnotetext{
5 Assuming the surface concentration of the piezoresistor is about $10^{20} \mathrm{~cm}^{-3}$.
}

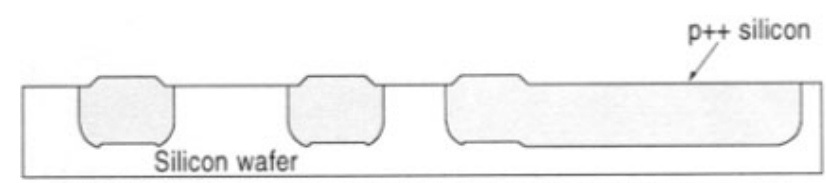

(a) Etch recess in silicon using RIE; selective deep boron diffusion

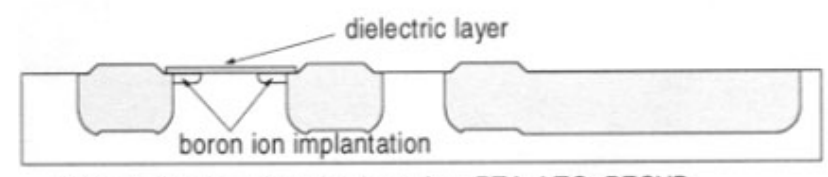

(b) Selective boron ion implantation; RTA; LTO; PECVD

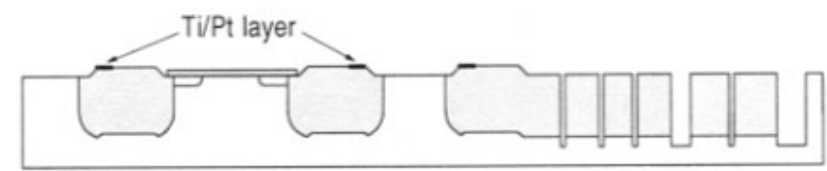

(c) Evapolate Ti/Pt layer; pattern microstructure using RIE; thinning

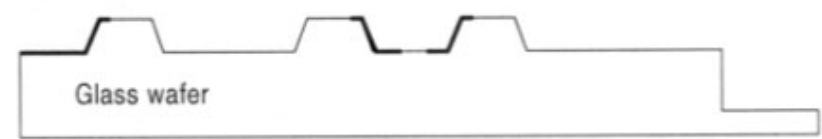

(d) Glass recess; evaporate metal; groove glass

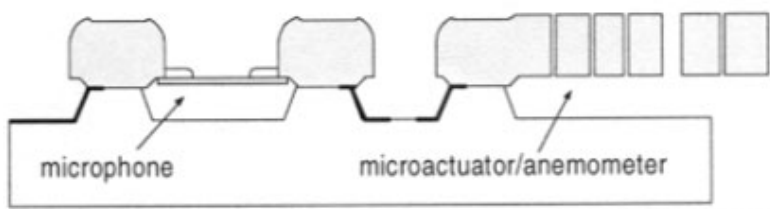

(e) Electrostatically bond silicon to the pattern glass wafer; EDP

Figure 3. The complete dissolved wafer process sequence for the fabrication of the microactuator/microsensor system.

by $1 \mu \mathrm{m}$ using RIE, except in those areas that will later be bonded to the glass substrate to anchor the actuators and sound detectors. This step also creates channels for equalizing the static pressure across the detector's diaphragm. Next, a selective deep boron diffusion $(\sim 15 \mu \mathrm{m})$ is performed at $1175^{\circ} \mathrm{C}$ for $15 \mathrm{~h}$. This step defines the area and the thickness of the microactuators and the rims (corners) of the sound detector. Because a small thickness is desired for the piezoresistors, boron ion implantation is chosen over shallow boron thermal diffusion, whose diffusion thickness is about $3 \mu \mathrm{m}$, which is ten times thicker than that obtained using ion implantation. A selective boron ion implantation at $40 \mathrm{keV}$ and a dose of $5 \times$ $10^{15} \mathrm{~cm}^{-2}$ is performed to define the piezoresistors. In order to activate the boron atoms, the wafer is annealed at $1000{ }^{\circ} \mathrm{C}$ for $30 \mathrm{~s}$. The details of boron ion implantation are described in [12]. After annealing, a $2260 \AA$ low temperature oxide layer is deposited at $420{ }^{\circ} \mathrm{C}$ followed by the deposition of a $1900 \AA$ PECVD nitride layer ${ }^{6}$ to form the diaphragm. The initial stress of the diaphragm can be reduced by varying the relative thickness of the nitride and the oxide films [13] to enhance the sensitivity of the sound detectors. The wafer is then patterned and metalized with a Ti/Pt layer in areas where lead transfers are to be made to the metal lines on the glass substrate. This improves the sound detector's sensitivity by reducing the contact resistance between the silicon and the

6 EDP attacks PECVD nitride film at a rate of about $200 \AA \mathrm{h}^{-1}$. The final thickness of this nitride layer is measured to be $1574 \AA$ after 90 min of EDP etching. 


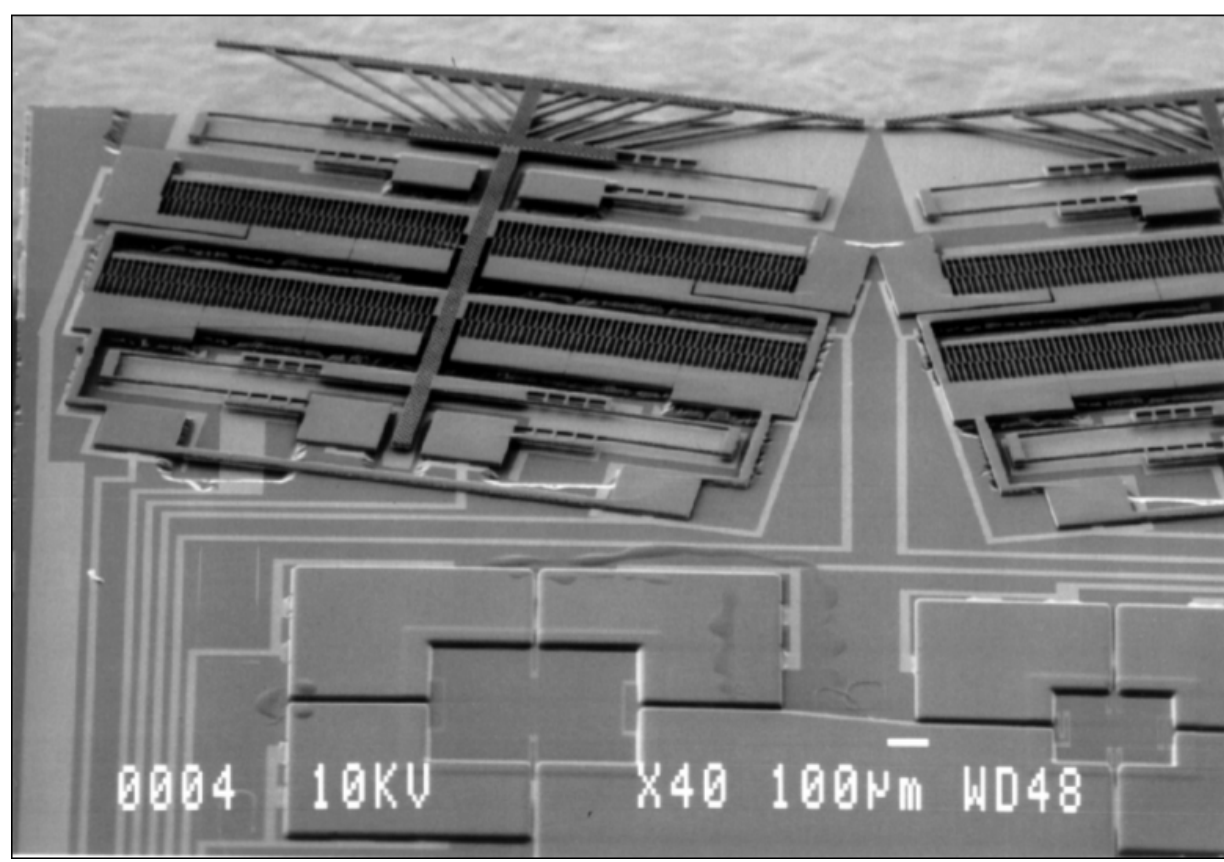

Figure 4. SEM view of a complete microactuator/microsensor system.

metal on the substrate [14]. The boron-diffused areas are then etched anisotropically using reactive ion etching (RIE) in a $\mathrm{SF}_{6} / \mathrm{O}_{2}$ gas plasma to pattern the fine microstructure of the actuators. This completes the silicon processing.

On the other hand, the glass substrate is patterned and recessed to a depth of about $20 \mu \mathrm{m}$ by a mixture of diluted hydrofluoric (HF) and nitric acids to create the bonding anchors. The recess in the glass substrate is created to avoid stiction of the silicon elements to the substrate and to reduce the damping effect on the sound detector's diaphragm and microactuators. The formation of such a gap is easily possible using the dissolved wafer process. Glass processing is completed after patterning $\mathrm{Ti} / \mathrm{Pt} / \mathrm{Au}$ interconnect lines on it. Finally, the silicon wafer is electrostatically bonded to the glass wafer, and the sandwich is immersed in EDP (ethylene diamine pyrocatechol, a concentration-dependent silicon etchant) to dissolve away the undoped silicon, leaving the $\mathrm{p}^{++}$silicon devices mounted on the glass substrate. The EDP temperature is $104{ }^{\circ} \mathrm{C}$, which is lower than the typical $110^{\circ} \mathrm{C}$ used in this process, for better control of the etch rate. The total EDP time is between $90 \mathrm{~min}$ and $2 \mathrm{~h}$. For an EDP etch at higher temperature or longer period than described above, the thin implanted $\mathrm{p}^{++}$layer could be attacked by EDP.

As mentioned before, the sound detectors need to be integrated with microactuators. Figure 4 shows a SEM view of a complete system containing two microactuators and two sound detectors [3]. The actuator details are not provided in this paper. Interested readers are referred to Huang et al [15].

\section{Sound detector performance}

The tests for the sound detectors are divided into two categories: static and dynamic. The equalization channel of the devices that are calibrated statically is sealed using vacuum epoxy to enable application of an adjustable static pressure on

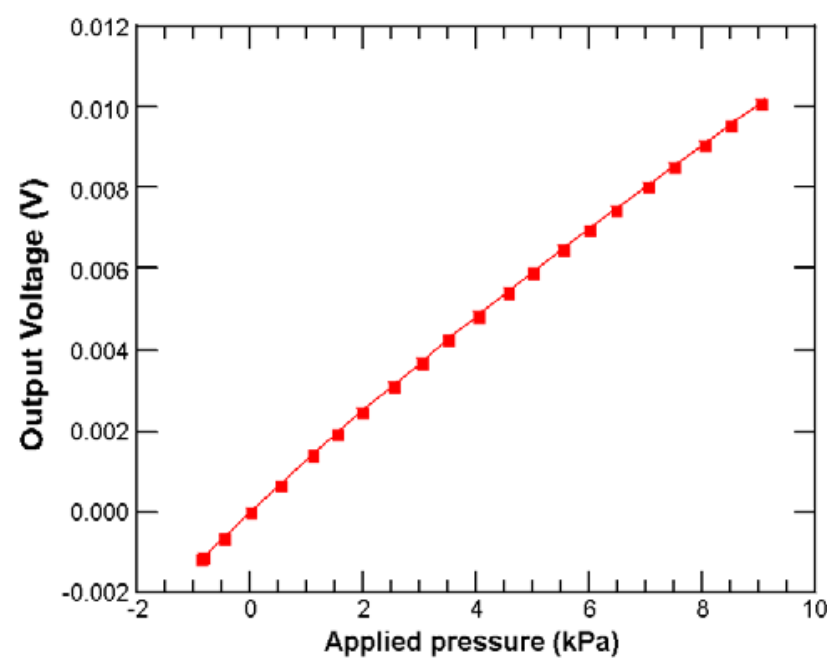

Figure 5. Static characteristics of a $710 \mu \mathrm{m}$ square sound detector.

the external side only of the sensor's diaphragm. These devices are also used in tests aimed at examining temperature effects and durability. On the other hand, devices that are characterized dynamically are exposed to an unsteady sound field without sealing of the equalization channel. Details and results of the static and dynamic tests follow.

\subsection{Static measurements}

Figure 5 shows the measured static response of a $710 \mu \mathrm{m}$ square sealed sound detector. The nominal impedance of the piezoresistors was $4 \mathrm{k} \Omega$ and the sensor was operated using a dc bridge excitation voltage of $10 \mathrm{~V}$, resulting in a nominal power dissipation of $25 \mathrm{~mW}$. The data in figure 5 were obtained by placing the sealed device into an adjustable-pressure pressurization/vaccum chamber. The results in figure 5 yield 


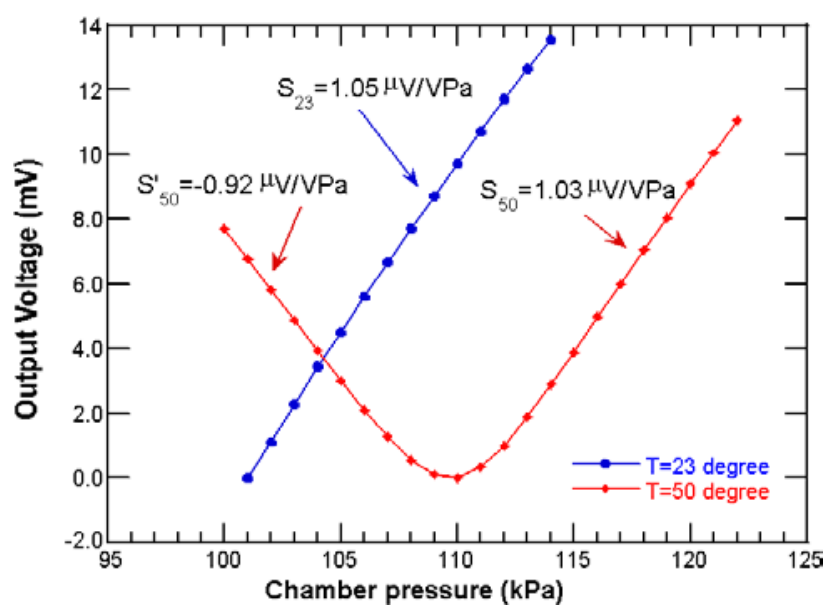

Figure 6. Temperature effect on the sensitivity of the new sensor.

a sensitivity of $1.1 \mu \mathrm{V} \mathrm{VPa}{ }^{-1}$, with a non-linearity of $2 \%$ over the full-scale range of $10 \mathrm{kPa}$. Figure 6 depicts the dependence of the sensor's static response on temperature. The measured temperature coefficient of sensitivity (TCS) is about $-705 \mathrm{ppm}{ }^{\circ} \mathrm{C}^{-1}$, which is less than the $-1330 \mathrm{ppm}{ }^{\circ} \mathrm{C}^{-1}$ reported in an earlier study [16]. This indicates the surface concentration of the piezoresistors is higher than the concentration of $10^{19} \mathrm{~cm}^{-3}$ reported in [16]. With the device sealed in ambient conditions, the pressure inside the chamber is $100 \mathrm{kPa}$ at room temperature. As the temperature increases by $10 \%$, the pressure inside the chamber increases by $10 \%$ to $110 \mathrm{kPa}$. As a result, there is a valley in the curve corresponding to measurements at $50{ }^{\circ} \mathrm{C}$, indicating an expansion of the trapped gas.

Finally, an overload pressure of $50 \mathrm{kPa}$ has been applied onto these devices without damaging the diaphragm. This demonstrates the robustness of the sensors that should allow them to be used in high-speed jets.

\subsection{Dynamic measurements}

Since the MEMS sensor was designed for screech noise measurements, it was desired to calibrate it in an acoustic field at a SPL of at least $100 \mathrm{~dB}$ over a wide frequency range. To generate this sound field, it was not possible to use a speaker due to an observed contamination of the MEMS output signal with noise from the audio amplifier. This was believed to be due to the lack of appropriate shielding and grounding of this first generation of MEMS sensors. Therefore, it was decided to produce the sound field via non-electrical means. To this end, an 'air siren' was constructed. The siren consisted of a $6 \mathrm{~mm}$ diameter air jet that was 'shuttered' periodically using a $150 \mathrm{~mm}$ diameter chopper wheel with slots cut along the circumference of the wheel. The passage of the slots in front of the jet created pressure pulsation at the slot-passing frequency, thus generating sound at a frequency that was adjustable by changing the disc rotation speed.

The minimum and maximum limits on the disc rotation rate were determined, respectively, by the lowest stable rotational speed and maximum driving voltage of the dc electric motor used to rotate the chopper disc. Four discs with different number of slots were used to generate sound at

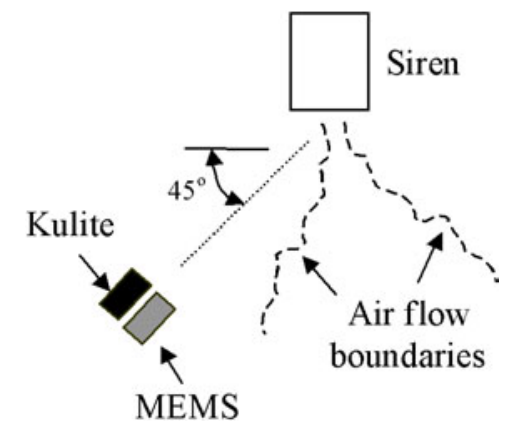

Figure 7. A schematic diagram of the dynamic calibration setup.

a frequency that varied from $100 \mathrm{~Hz}$ to $10 \mathrm{kHz}$. The location of sensor calibration within the siren's sound field was chosen to be outside the boundaries of the air jet flow. This was done to ensure that the output of the sensor was due to the acoustic and not the hydrodynamic pressure fluctuations. The resulting sensor location was about $0.6 \mathrm{~m}$ (or 100 jet diameters) away from the jet exit at an angle of $45^{\circ}$ from the jet axis, as seen in figure 7.

To obtain the MEMS sensor's dynamic response, it was placed next to a commercial pressure transducer with known characteristics in the sound field of the siren at the calibration location. The 'reference' transducer was a Kulite model XCS$062-5 \mathrm{G}$ with a nominal sensitivity of $0.2 \mu \mathrm{V} \mathrm{VPa}-1$ and a flat frequency response up to about $20 \mathrm{kHz}$. Although larger in size, the Kulite sensor also utilizes a silicon diaphragm with four piezoresistors arranged in a Wheatstone bridge to detect the deflection of the diaphragm under the action of the measured pressure.

Due to the similar principle of operation of the MEMS and the Kulite sensors, the excitation voltage and signal conditioning of both sensors were achieved using AD 1B31AN strain gauge signal conditioner from analog devices. The Kulite output was amplified by a factor of 2500 using the signal conditioner, while the MEMS sensor signal was amplified by a factor of 2000. The signals were further low-pass filtered at the Nyquist frequency to eliminate aliasing in data recording.

The outputs of the Kulite and MEMS sensors were acquired simultaneously at a sampling rate of ten times the sound frequency. The measurements were used to obtain the power spectra of the MEMS and Kulite voltage time series. 400 records of 2048 points were used to obtain each spectrum. This resulted in a spectrum frequency resolution of $0.5 \%$ of the sound frequency. The random uncertainty in the spectral estimate was approximately $5 \%$, based on 400 records and assuming Gaussian random variation in the measurements. The sensitivity of the MEMS sensor at a given frequency was determined from the equation

$$
K_{\mathrm{MEMS}}=K_{\mathrm{Kulite}} \sqrt{\frac{E_{\mathrm{vv}, \mathrm{MEMS}}}{E_{\mathrm{vv}, \text { Kulite }}}}
$$

where $K_{\text {Kulite }}$ is the Kulite sensitivity in $\mathrm{mV} \mathrm{Pa}^{-1}$ and $E_{\mathrm{vv}, \mathrm{MEMS}}$ and $E_{\mathrm{vv}, \text { Kulite }}$ represent the energy contained in the voltage spectrum peak at the frequency of the siren sound for the MEMS and Kulite, respectively. The energy values were obtained from integration of the spectra over a narrow frequency range around the sound frequency. The integration 


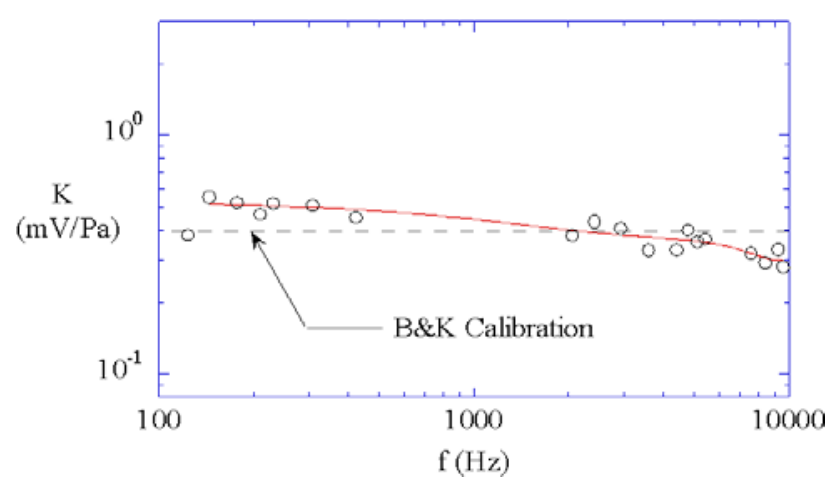

Figure 8. Calibration of $\mathrm{B} \& \mathrm{~K}$ microphone using the siren calibration setup.

was necessary due to some jitter in the motor rotational speed observed during data recording. This jitter, however, was less than $4 \%$ for all frequencies

Prior to calibration of the MEMS sensors in the siren's sound field, it was desired to verify the calibration process. Therefore, a $1 / 8^{\prime \prime} \mathrm{B} \& \mathrm{~K}$ microphone with known sensitivity was used in place of the MEMS sensor and calibrated against the Kulite in the manner outlined above. The results of the calibration are compared to the $\mathrm{B} \& \mathrm{~K}$ calibration provided by the manufacturer in figure 8 . As seen from the figure, the calibration procedure provides a good estimate of the $\mathrm{B} \& \mathrm{~K}$ sensitivity. However, the procedure seems to produce a systematic error, whereby the microphone sensitivity is overestimated at low frequencies and underestimated at high ones, giving a false impression of sensitivity attenuation with increasing frequency. The deviation, which is about $3 \mathrm{~dB}$ at both frequency ends, may be caused by sound directivity of the siren and/or diffraction by the package of the sensors.

Since the observed attenuation represents a systematic error, it can be accounted for when calibrating the MEMS sensors. To accomplish this, a least-squares curve fit was obtained for the B\&K calibration data (except for the one at the lowest frequency which seemed to depart from the observed trend) and employed to develop an equation for a correction factor as a function of frequency. The frequency-dependent correction factor was obtained by dividing the curve fit (shown by a solid line in figure 8 ) by the known B\&K sensitivity. Subsequently, the MEMS sensor sensitivity at each frequency was divided by the correction factor value at that frequency to remove the attenuation trend associated with the calibration process.

Another important check on the MEMS calibration procedure is to verify that the MEMS output due to the acoustic measurements is much larger than the electrical noise level. To achieve this, the spectra measured by the MEMS, with and without the acoustic field, were compared. It was possible to turn off the acoustic field by simply shutting down the air supply to the siren while the motor was running. A sample of the results at a motor rotation speed of $6000 \mathrm{rpm}$ is shown in figure 9. Inspection of the figure shows that while the air and motor are turned on, a large acoustic peak exists at $5.5 \mathrm{kHz}$. The magnitude of this peak is at least four orders of magnitude larger than the background noise observed when shutting off the air supply only or both the air supply and motor. This also demonstrates that the peak in the spectrum at the acoustic

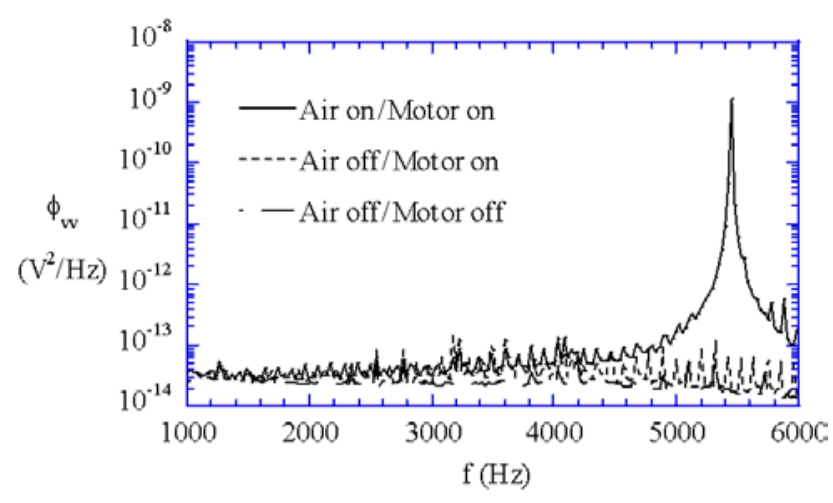

Figure 9. Signal to noise level at the calibration location.
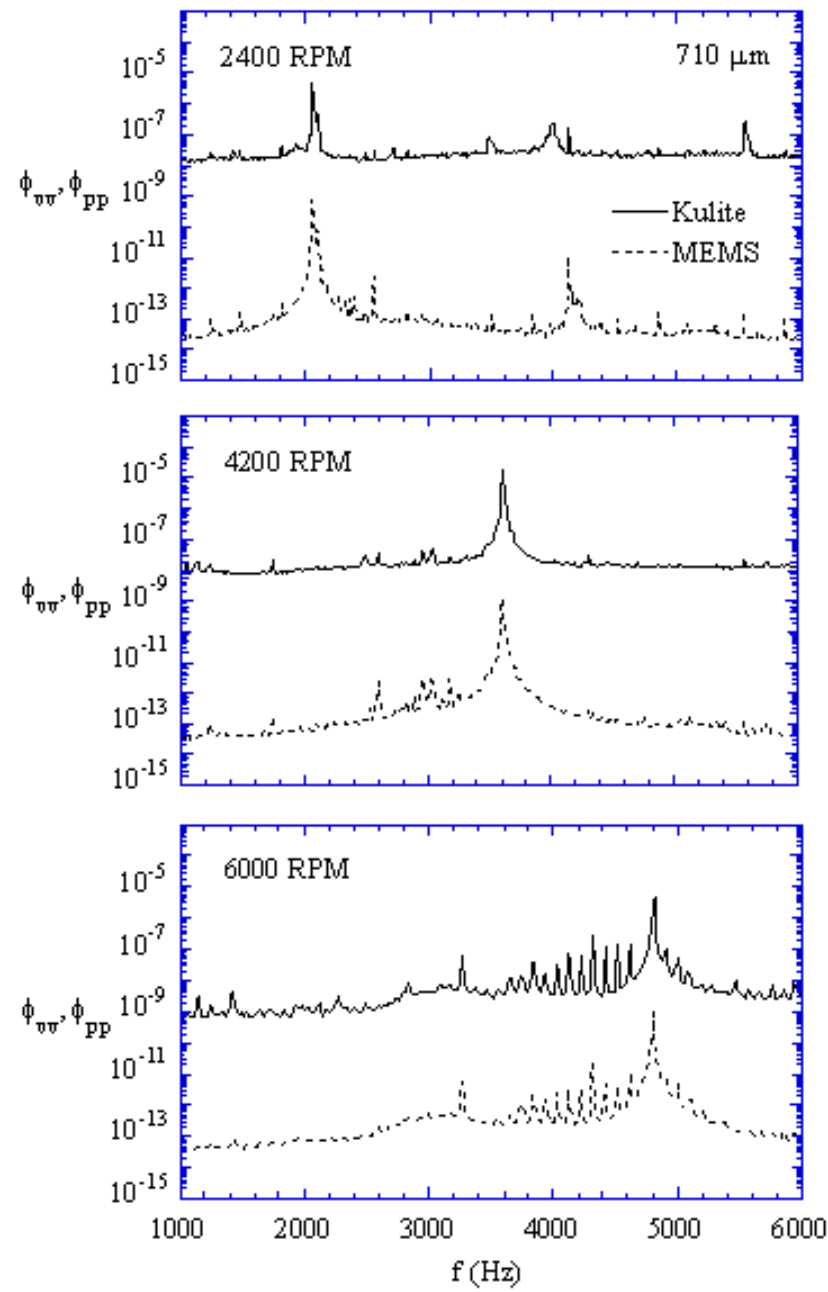

Figure 10. Sample spectra measured by the $710 \mu \mathrm{m}$ MEMS and Kulite sensors (units of $\mathrm{V}^{2} \mathrm{~Hz}^{-1}$ and $\mathrm{kPa}^{2} \mathrm{~Hz}^{-1}$, respectively).

frequency is in fact due to the acoustic field, and not due to any electrical noise induced by the motor. As mentioned earlier, induced electrical noise was a problem when attempting to use an audio amplifier coupled to a speaker to conduct the calibration.

The voltage spectra measured using a $710 \mu \mathrm{m}$ sensor for three different rotational speeds of the siren are provided in figure 10. For comparison purpose, the pressure spectra measured using the Kulite under identical conditions as the 


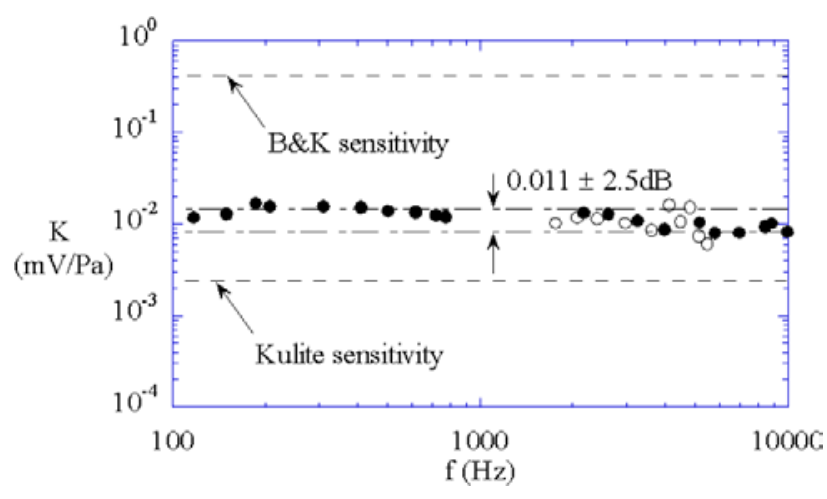

Figure 11. Response of the MEMS sensor compared to the Kulite and $\mathrm{B} \& \mathrm{~K}$.

MEMS are also provided in the figure. It is seen from figure 10 that the shape of the spectra obtained from the MEMS and Kulite sensors is the same. It should be noted that the height of the spectrum peak relative to the background noise (i.e., the flat part of the spectrum) might be used to assess the signal to noise ratio of the sensors. Inspection of figure 10 shows that this peak height above the noise level for the MEMS sensor is about two to three times higher than that for the Kulite. In fact, if the background spectrum level of the MEMS device (approximately $10^{-14} \mathrm{~V}^{2} \mathrm{~Hz}^{-1}$, as seen in figure 10) is converted into a noise-equivalent pressure spectrum using the sensitivity value obtained in section 3.1 , a value of approximately $10^{-10} \mathrm{kPa}^{2} \mathrm{~Hz}^{-1}$ is obtained. This is one to two orders of magnitude smaller than the background spectrum level of the Kulite, which is about $10^{-8} \mathrm{kPa}^{2} \mathrm{~Hz}^{-1}$ in the top two plots in figure 10 and $10^{-9} \mathrm{kPa}^{2} \mathrm{~Hz}^{-1}$ in the lowermost one.

Figure 11 provides the full dynamic calibration results for the MEMS sensor. For reference, the sensitivity of the Kulite and B\&K microphones is also provided in the figure. The filled circles represent the current calibration results for the $710 \mu \mathrm{m}$ sensor, while the open symbols correspond to a calibration of the same sensor obtained 2 months earlier over a narrower frequency range.

The results in figure 11 depict a good agreement between the open and filled circles. This agreement demonstrates the stability of the developed MEMS transducers. The variation in the sensitivity over the entire frequency range is within $\pm 2.5 \mathrm{~dB}$ around the static sensitivity value of $0.011 \mathrm{mV} \mathrm{Pa}^{-1}$ (or $1.1 \mu \mathrm{V} \mathrm{VPa}^{-1}$ for the bridge excitation voltage of $10 \mathrm{~V}$ used in the tests, as obtained in section 3.1). It is noteworthy that this sensitivity value is about five times larger than that of the commercial Kulite sensor. With the current calibration procedure, it is not possible to verify whether this high sensitivity is maintained beyond $10 \mathrm{kHz}$. However, the frequency range examined here includes the jet screech noise frequencies that the sensor is intended to measure (as discussed in section 1).

All results indicate that most of the fundamental requirements for measuring the screech noise have been successfully met by this first generation of piezoresistive microphones. These include linearity, low temperature sensitivity, robustness, sufficiently high sensitivity, wide bandwidth and signal to noise ratio that is at least at par with commercially available silicon-based sensors. Issues to address in future generations of the sensor should include engineering of appropriate provisions for signal shielding and grounding to minimize device sensitivity to extraneous noise, as well as examination of possible cross-talk between actuator driving signal and sensor output.

\section{Conclusion}

A new MEMS piezoresistive sound detector has been developed and characterized. The sensor is the first of this type to be fabricated using the dissolved wafer process. Furthermore, the new device capitalizes on the high piezoresistive coefficient and low temperature dependence of highly boron-doped single-crystal silicon.

The performance of the new sensor was verified using static and dynamic tests. The results yield a static sensitivity of $1.1 \mu \mathrm{V} \mathrm{VPa}{ }^{-1}$, non-linearity of $2 \%$ over the full-scale pressure range of $10 \mathrm{kPa}$, and a temperature sensitivity of $-705 \mathrm{ppm}{ }^{\circ} \mathrm{C}^{-1}$. It is interesting to note that the documented sensitivity is significantly larger than commercial siliconbased piezoresistive pressure sensors. On the other hand, the frequency response of the device has a flat sensitivity (within $\pm 2.5 \mathrm{~dB}$ ) over the frequency range extending from 100 to $10000 \mathrm{~Hz}$.

\section{Acknowledgments}

The authors are grateful for the Air Force Office of Scientific Research support of this work, monitored by Dr Jim McMichael and Dr Mark Glauser under grant numbers F49620-94-0184 and F49620-96-029. Additionally, access to laboratory resources at Illinois Institute of Technology provided by Dr Hassan Nagib to complete this work is greatly appreciated.

\section{References}

[1] Scheeper P R, Van der Donk A G H, Oltheruis W and Bergveld P 1994 A review of silicon microphones Sensors Actuators A 44 1-11

[2] Huang C, Papp J, Najafi K and Nagib H 1996 A microactuator system for the study and control of screech in high speed jets Proc. 9th Annual Int. Workshop on MEMS pp 19-24

[3] Huang C, Najafi K, Alnajjar E, Christophorou C, Naguib A and Nagib H 1998 Operation and testing of electrostatic microactuators and micromachined sound detectors for active control of high speed flows Proc. 11th Annual Int. Workshop on MEMS (January 1998) pp 81-6

[4] Tam C 1991 Jet noise generated by large-scale coherent motions Aeroacoustics of Flight Vehicles: Theory and Practice vol 1 ed H H Hubbard NASA Reference Publication 1258 pp 311-90

[5] Kälvesten E, Löfdahl L and Stemme G 1995 Small piezoresistive silicon microphones specially designed for the characterization of turbulent gas flows Sensors Actuators A 46-7 151-5

[6] Schellin R and Hess G 1992 A silicon subminiature microphone based on piezoresistive polysilicon strain gauges Sensors Actuators A 32 555-9

[7] Sheplak M, Breuer K S and Schmidt M A 1998 A wafer-bonded, silicon-nitride membrane microphone with dielectrically-isolated, single-crystal silicon piezoresistors Proc. Solid-State Sensor and Actuator Workshop (Hilton Head, SC, June 1998) 
[8] Kanda Y 1991 Piezoresistance effect of silicon Sensors Actuators A 28 83-91

[9] Tufte O N and Stelzer E L 1963 Piezoresistive properties of silicon diffused layers J. Appl. Phys. 34 313-8

[10] Cho S T 1991 An ultrasensitive silicon pressure-based microflow sensor PhD dissertation the University of Michigan

[11] Gianchandani Y B and Najafi K 1992 A bulk silicon dissolved wafer process for microelectromechanical systems IEEE/ASME J. Microelectromech. Syst. 1 77-85

[12] Huang C and Najafi K 2001 Fabrication of ultra-thin $\mathrm{P}^{++}$ silicon microstructures using ion implantation and boron etch-stop J. Microelectromech. Syst. 10 532-7
[13] Zhang Y 1994 Non-planar diaphragm structures for high performance silicon pressure sensors $\mathrm{PhD}$ dissertation the University of Michigan

[14] Robertson J K 1996 An electrostatically-actuated integrated microflow controller $\mathrm{PhD}$ dissertation the University of Michigan

[15] Huang C, Christophorou C, Najafi K, Naguib A and Nagib H 2001 An electrostatic microactuator system for application in high speed jets IEEE/ASME J. Microelectromech. Syst. 11 222-35

[16] Kim S-C and Wise K D 1983 Temperature sensitivity in silicon piezoresistive pressure transducers IEEE Trans. Electron Devices 30 802-10 